IRSTI 27.35.14

\author{
${ }^{1,2^{*}}$ A.S. Zhumali, ${ }^{1}$ B.A. Satenova, ${ }^{1,2}$ O.L. Karuna \\ ${ }^{1}$ Al-Farabi Kazakh National University, Almaty, Kazakhstan \\ ${ }^{2}$ National Engineering Academy, Almaty, Kazakhstan \\ *e-mail: ainura.z89@gmail.com
}

\title{
Lattice Boltzmann method simulation of thermal flow dynamics in a channel
}

\begin{abstract}
The objective of this paper is the simulation of thermal flow dynamics in a channel. The mathematical model in a two-dimensional formulation is described by Navier-Stokes equations, continuity and temperature equations. For the numerical simulation of the problem the Lattice Boltzmann method applying the D2Q9 model is used. The validity of this method is tested by comparing the numerical solution to the analytical solution of the planar channel flow and error rates are calculated for various sizes of the computational grid. The test problem of thermal Poiseuille flow in the channel was solved to deactivate the correctness of the developed algorithm. Very good agreement between the exact and numerical solution of this problem is shown.
\end{abstract}

Key words: The lattice Boltzmann method, thermal flow dynamics, Poiseuille flow.

\section{Introduction}

Thermal flows play an important role in the flow dynamics. Recently, there has been an effort to increase the capability of the lattice Boltzmann method in order to solve for fluid flows including heat transfer $[1,2]$. A detailed analysis can be found in [3].

Generally, the thermal lattice Boltzmann model (TLBE) can be divided into several categories [4]. The first is the multispeed scheme, the second is the double distribution function (DDF) scheme and the last is the hybrid thermal lattice Boltzmann equation (HTLBE) scheme [3]. The multi-speed scheme is a plain extension of the Boltzmann isothermal models with a lattice, in which only the velocity distribution function is affected. In double distribution function scheme, different distribution functions are used, one for the velocity field and the other for the temperature field or internal energy. The main advantage of the DDF scheme compared to the multi-speed scheme is to increase the numerical stability, and therefore it is widely used. The hybrid computational scheme combines the LBE and Finite difference (FD) or Finite volume (FV) methods [5]. In this paper we use DDF scheme.
The goal of this paper is the numerical implementation of thermal flow dynamics in a channel in a two-dimensional case. With the help of LBE method the profiles of velocities and temperature at different values of parameters in the system of differential equations and at different time instants are investigated.

\section{Statement of the problem}

In this paper we considered 2-D thermal flow in planar channel. The flow driven by a body force. We set cold temperature at the bottom wall and hot temperature at the top wall of the channel (Figure 1).

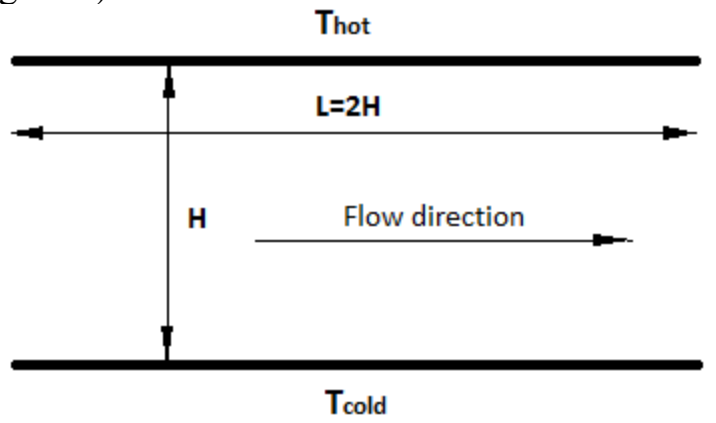

Figure 1 - The considered area 
The governing equations can be written as [6]:

$$
\begin{gathered}
\frac{\partial u_{i}}{\partial t}+\frac{\partial}{\partial x_{j}}\left(u_{i} u_{j}\right)=-\frac{1}{\rho} \frac{\partial p}{\partial x_{i}}+v \frac{\partial^{2} u_{i}}{\partial x_{j} \partial x_{j}}+F_{x}, \\
\frac{\partial u_{i}}{\partial x_{i}}=0 \\
\frac{\partial T}{\partial t}+\frac{\partial}{\partial x_{j}}\left(u_{j} T\right)=k \frac{\partial^{2} T}{\partial x_{j} \partial x_{j}} .
\end{gathered}
$$

Here, $\rho$ is the linear function of the temperature $T$ and $F_{x}$ is the body force

$$
\begin{gathered}
\rho=\rho_{0}-\rho_{0} \beta\left(T-T_{0}\right), \\
F_{x}=8 \rho_{0} v u_{\text {max }} / H^{2},
\end{gathered}
$$

where $\rho_{0}$ is the average density, $T_{0}$ is the average temperature, $\beta$ is the coefficient of thermal expansion.

We assume that at the initial time, the velocity and temperature in the channel are zero. Periodic boundary conditions are used at the channel inlet and outlet for $u_{1}, u_{2}, T, p$. And the following boundary conditions are applied on channel walls

$$
\begin{gathered}
u_{1}\left(x_{1}, x_{2}=0, t\right)=u_{1}\left(x_{1}, x_{2}=H, t\right)= \\
=u_{2}\left(x_{1}, x_{2}=0, t\right)=u_{2}\left(x_{1}, x_{2}=H, t\right)=0, \\
T\left(x_{1}, x_{2}=0, t\right)=T_{\text {cold }}, \\
T\left(x_{1}, x_{2}=H, t\right)=T_{\text {hot }} .
\end{gathered}
$$

\section{Numerical method}

The lattice Boltzmann equation (LBE) method is a discrete model of a continuous medium. Currently, the LBE method may well compete with traditional methods of computational hydrodynamics, and in some areas (flows in a porous medium, multiphase and multicomponent flows) it has significant advantages [7-9]. By this method an intermediate scale model is used to simulate fluid flow. It applies simulation of the motion of fluid particles in order to capture the macroscopic parameters of the fluid. The area is discretized by uniform cartesian cells.
Each cell contains a fixed number of distribution functions, which represent the number of fluid particles moving in these discrete directions. Depending on the dimension and the number of directions of velocity, there are various models that can be used. In the present study, a two-dimensional flow and a two-dimensional square lattice with nine discrete velocities (D2Q9 model) are examined. For each velocity vector, the value of the distribution function is stored. In the D2Q9 model (Figure 2), the velocities are calculated using the formulas

$$
\begin{gathered}
e_{0}=(0,0), \quad e_{1}=(1,0), \quad e_{2}=(0,1), \\
e_{3}=(-1,0), e_{4}=(0,-1), \\
e_{5}=(1,1), \quad e_{6}=(-1,1), \\
e_{7}=(-1,-1), e_{8}=(1,-1),
\end{gathered}
$$

where $c=\Delta x / \Delta t$ and $k-$ lattice velocity direction.

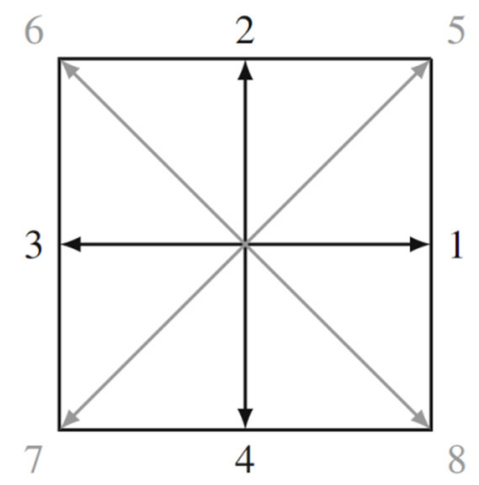

Figure 2 - D2Q9-model

Distribution functions are calculated by solving the lattice Boltzmann equation, which is a special discretization of the Boltzmann kinetic equation. After introducing the Bhatnagar - Gross - Crook approximation, we can formulate the Boltzmann equation in the form [10]

$$
f_{i}\left(x+e_{i} \Delta t, t+\Delta t\right)=f_{i}(x, t)+\frac{\Delta t}{\tau}\left[f_{i}^{e q}(x, t)-f_{i}(x, t)\right]
$$

where $\Delta t$ denotes the lattice time step, $e_{i}$ is the discrete lattice velocity in the direction $i, \tau$ denotes the lattice relaxation time, $f_{i}^{e q}$ is the equilibrium distribution function. The equilibrium distribution functions are calculated by the formula 


$$
f_{i}^{e q}=\omega_{i} \rho\left[1+\frac{e_{i} \cdot \vec{u}}{c_{s}^{2}}+\frac{1}{2} \frac{\left(e_{i} \cdot \vec{u}\right)^{2}}{c_{s}^{4}}-\frac{1}{2} \frac{\vec{u}^{2}}{c_{s}^{2}}\right]
$$

where the values of the weight coefficients $\omega_{i}$ are as follows:

$$
\omega_{i}= \begin{cases}4 / 9, & i=0 \\ 1 / 9, & i=1,2,3,4, \\ 1 / 36, & i=5,6,7,8,\end{cases}
$$

and $c_{S}=c / \sqrt{3}$ is the lattice speed of sound.

The macroscopic variables for the density and velocity of a fluid are calculated as the first two moments of the distribution functions for each cell:

$$
\rho=\sum_{i=0}^{8} f_{i}, \quad \vec{u}=\frac{1}{\rho} \sum_{i=1}^{8} f_{i} e_{i} .
$$

For the temperature field, the distribution $g$ is

$$
\begin{gathered}
g_{i}\left(x+e_{i} \Delta t, t+\Delta t\right)= \\
=g_{i}(x, t)+\frac{\Delta t}{\tau_{g}}\left[g_{i}^{e q}(x, t)-g_{i}(x, t)\right] .
\end{gathered}
$$

The equilibrium distribution functions for the temperature field are determined by the formulas:

$$
g_{i}^{e q}=\omega_{i} T\left[1+\frac{e_{i} \cdot \vec{u}}{c_{s}^{2}}+\frac{1}{2} \frac{\left(e_{i} \cdot \vec{u}\right)^{2}}{c_{s}^{4}}-\frac{1}{2} \frac{\vec{u}^{2}}{c_{s}^{2}}\right]
$$

The temperature field is calculated by the formula

$$
T=\sum_{i=0}^{8} g_{i} .
$$

As the no-slip boundary condition in fixed walls of channel the mid-link bounce back scheme is used $[6,11]$. According to the scheme, the wall boundary is located half lattice away from the boundaries of the fluid nodes. At the boundary node, the outward post-streaming distribution is equal to the inward pre-streaming distribution:

$$
f_{i}^{-}\left(x_{B}, t+\Delta t\right)=f_{i}\left(x_{B}, t\right),
$$

where $x_{B}$ is the coordinates of the boundary node, $f_{i}$ is the pre-streaming distribution function with discrete velocity $e_{i}$, which points into the boundary. $f_{i}^{-}$is the post-streaming distribution function in the direction opposite to $e_{i}$. For the constant temperature boundary, the distribution function can be obtained as

$$
g_{i}^{-}\left(x_{B}, t+\Delta t\right)=-g_{i}\left(x_{B}, t\right)+2 \omega_{i} T_{B},
$$

where $T_{B}$ is the temperature of the top or bottom wall and $\omega_{i}$ is the weight coefficients.

Algorithm for applying the lattice Boltzmann method:

1. Discretization of the physical domain and nondimensionalization of the related parameters;

2. Choice of simulation parameters;

3. Domain initialization;

4. Collision step;

5. Application of the boundary conditions;

6. Streaming step;

7. Calculation of the macroscopic parameters;

8. Verification of convergence criteria. If criterion is met, then end of routine, else go to 4.

\section{Analytical solution and numerical results}

In order to check the developed algorithm for solving the problem of thermal flow, the problem of two-dimensional Poiseuille flow in channel is solved.

Table 1 sets the parameters for calculating the test problem. The simulation was performed with different sizes of the computational domain: $N_{x} \times N_{y}=100 \times 50,200 \times 100$. The maximum velocity $u_{\max }$ in the channel and the sound speed $c_{s}$ are 0.1 and 0.5773 , respectively. Kinematic viscosity $v=9.021 \cdot 10^{-3}$. The height of the channel $H=1$. The Reynolds number $\operatorname{Re}=u_{\max } \cdot H / v \approx 10$. In the present study the Prandtl number $\operatorname{Pr}=\frac{v}{k}=0.7$. Relaxation parameters are defined as [12] $\tau=\frac{v}{c_{S}^{2} \cdot \Delta t}+0.5$ and $\tau_{g}=\frac{k}{2 c_{s}^{2} \Delta t}+0.5$.

Along the $X$ axis the constant pressure difference is maintained: 


$$
\frac{\Delta p}{x_{\text {out }}-x_{\text {in }}}=\frac{8 \eta u_{\max }}{\left(y_{\text {top }}-y_{\text {bot }}\right)^{2}}
$$

where $\Delta p$ is the pressure difference, $\Delta p=p_{\text {out }}-p_{\text {in }}, p_{\text {out }}$ and $p_{\text {in }}$ are the pressure at the outlet and at the inlet of the channel, respectively, $\eta$ is the dynamic viscosity, $x_{\text {out }}$ and $x_{i n}$ are the outlet and the inlet boundaries, respectively, $y_{\text {top }}$ and $y_{\text {bot }}$ are the top and the bottom walls boundaries, respectively.

Table 1 - Simulation parameters

\begin{tabular}{|c|c|}
\hline Parameters & \\
\hline scaling factor, scale & scale $=1: 2$ \\
\hline number of points along the $x$ axis $N_{x}$ & $N_{x}=100 \cdot$ scale \\
\hline number of points along the $y$ axis, $N_{y}$ & $N_{y}=50 \cdot$ scale \\
\hline relaxation parameter, $\tau$ & $\tau=\sqrt{3 / 16}+0.5$ \\
\hline maximum velocity in a channel, $u_{\max }$ & $u_{\max }=0.1 /$ scale \\
\hline kinematic viscosity, $v$ & $v=(2 \tau-1) / 6$ \\
\hline Reynolds number, $\operatorname{Re}$ & $\operatorname{Re}=10$ \\
\hline Prandtl number, $\operatorname{Pr}$ & $\operatorname{Pr}=0.7$ \\
\hline channel outlet pressure, $p_{\text {out }}$ & $p_{\text {out }}=1$ \\
\hline
\end{tabular}

The analytical solutions for the velocity and the temperature fields are calculated as $[12,13]$ :

$$
\begin{gathered}
u_{\text {exact }}(y)=u_{\max }\left(1-\frac{y^{2}}{L^{2}}\right), \\
T_{\text {exact }}(y)=T_{b o t}+\frac{\left(T_{\text {top }}-T_{b o t}\right)}{H} y+ \\
+\frac{1}{3} \operatorname{Pr} u_{\max }^{2}\left[1-\left(\frac{2 y}{H}-1\right)^{4}\right] .
\end{gathered}
$$

The comparison of the exact solution with the results of the numerical solution is observed in Figures $3-5$.

$L_{1}$ and $L_{2}$ norms of error were calculated by the following formulas:

$$
\begin{gathered}
\varepsilon_{L_{1}}(t)=\frac{\sum_{x_{1}, x_{2}}\left|q_{n}\left(x_{1}, x_{2}, t\right)-q_{b}\left(x_{1}, x_{2}, t\right)\right|}{\sum_{x_{1}, x_{2}}\left|q_{b}\left(x_{1}, x_{2}, t\right)\right|}, \\
\varepsilon_{L_{2}}(t)=\frac{\sqrt{\sum_{x_{1}, x_{2}}\left|q_{n}\left(x_{1}, x_{2}, t\right)-q_{b}\left(x_{1}, x_{2}, t\right)\right|^{2}}}{\sum_{x_{1}, x_{2}}\left|q_{b}\left(x_{1}, x_{2}, t\right)\right|^{2}} .
\end{gathered}
$$

where the index $n$ means numerical solution and the index $b$ means analytical solution.

Increasing the grid resolution helps to reduce the error norms. If we assume that the error norm is known for different grid sizes and their ratio of the sizes of each grid to the initial one is $\mathrm{m}$, then we can determine the order of accuracy using the following formula:

$$
n(t)=\log _{m}\left(\frac{\varepsilon_{0}(t)}{\varepsilon_{m}(t)}\right)
$$

In the test problem, the error norms were calculated at time $t \cdot v / L^{2}=1$. The accuracy orders of the numerical algorithm depending on the grid size are presented in table 2 .

Figure 3 shows the predicted cross-sectional profile in the force-driven channel flow for velocity and comparison between LBM simulation and analytical solution. Solid line is the analytical solution and the symbol is the numerical result.

Table 2 - The accuracy orders of $L_{1}$ and $L_{2}$ of the velocity, depending on the grid size at $t \quad v \cdot / L^{2}=1$

\begin{tabular}{|c|c|c|c|c|}
\hline Grid size & $u\left(L_{1}\right)$ & Order of accuracy, $n$ & $u\left(L_{2}\right)$ & Order of accuracy, $n$ \\
\hline $100 \times 50$ & $4.6600 \times 10^{-4}$ & 3.8978 & $6.8264 \times 10^{-2}$ & 1.9489 \\
\hline $200 \times 100$ & $1.6972 \times 10^{-4}$ & 3.8175 & $4.1198 \times 10^{-3}$ & 1.9087 \\
\hline
\end{tabular}


Also, Figure 4 shows the temperature crosssectional variation at different time instants in comparison with the analytic solution. Solid lines are the numerical results and the symbol is the analytical solution. And Figure 5 demonstrates the temperature cross-sectional profiles in comparison with the analytic solution for different Prandtl numbers. Here, solid lines are the analytical solutions and the symbols are the numerical results. As can be seen from the figures, the numerical results agree well with the analytical solutions. The general results in terms of streamwise temperature and velocity for time instants $t=0.1$ and $t=1$ are shown in Figures 6 and 7, respectively.

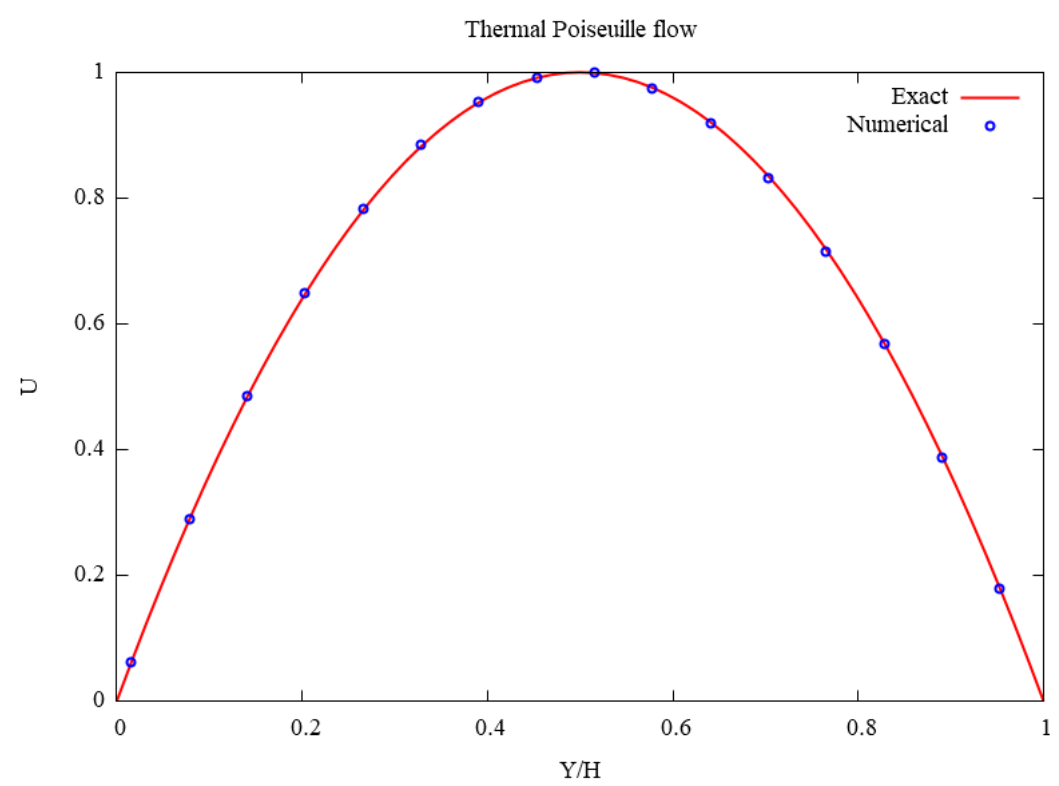

Figure 3 - Velocity profile of a 2D Poiseuille flow.

Comparison of the exact solution with the result of the numerical solution at

$$
T_{\text {top }}=1, T_{b o t}=0, \operatorname{Pr}=0.7, u_{\max }=0.1 \text {. }
$$

Thermal Poiseuille flow

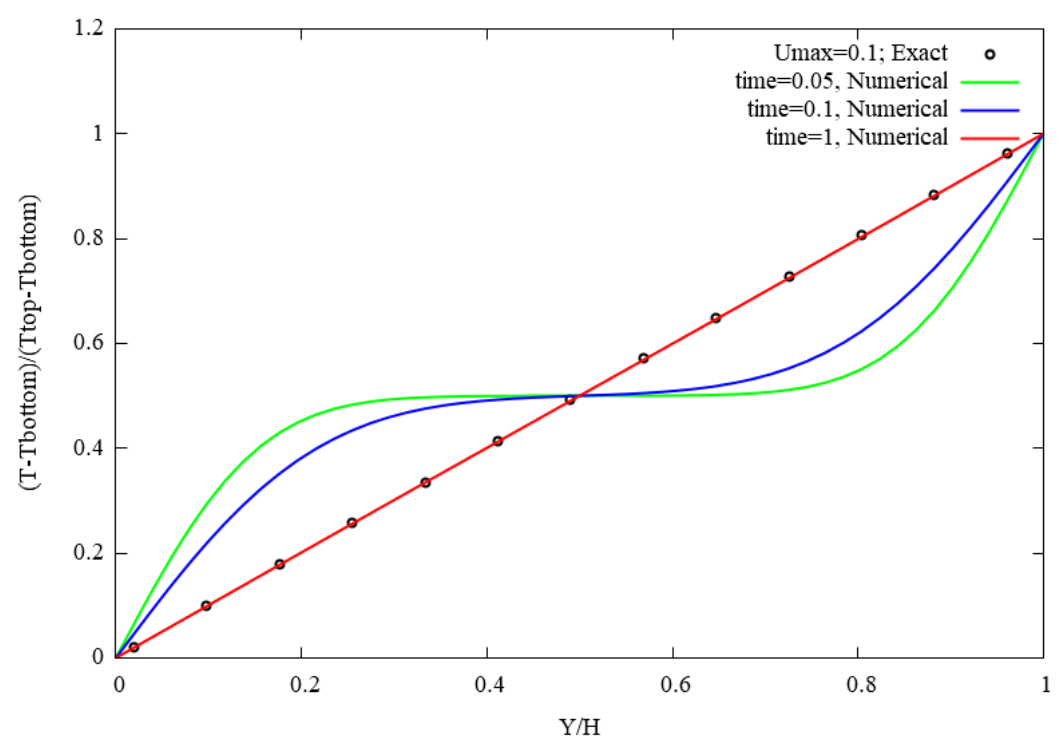

Figure 4 - Temperature variation of a 2D Poiseuille flow.

Comparison of the exact solution with the result of the numerical solution at $T_{\text {top }}=1, T_{\text {bot }}=0, \operatorname{Pr}=0.7, u_{\max }=0.1$ 


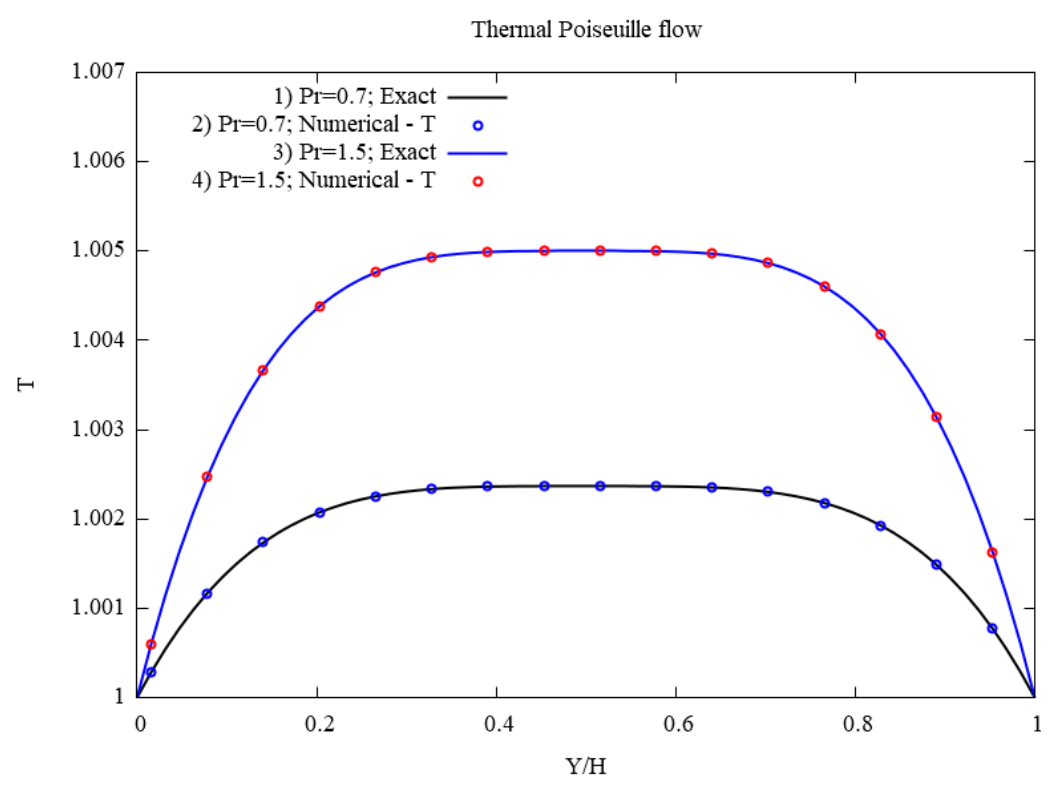

Figure 5 - Temperature profiles of a 2D Poiseuille flow.

Comparison of the exact solution with the result of the numerical solution at $T_{\text {top }}=1, T_{\text {bot }}=1, \quad \operatorname{Pr}=0.7$ and $\operatorname{Pr}=1.5, \quad u_{\max }=0.1$

T: $\quad 0.05 \quad 0.30 \quad 0.55 \quad 0.80$

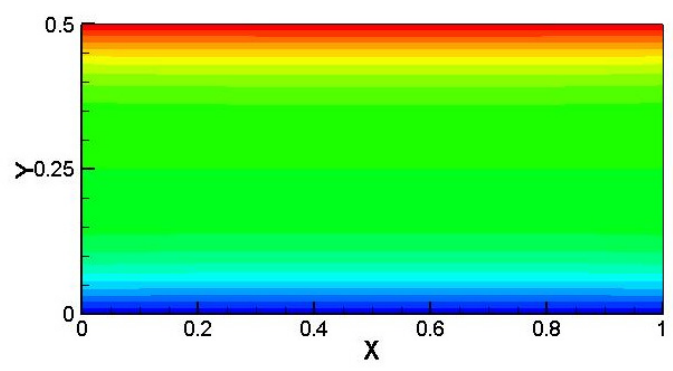

(a)
T: $\quad 0.05 \quad 0.30 \quad 0.55 \quad 0.80$

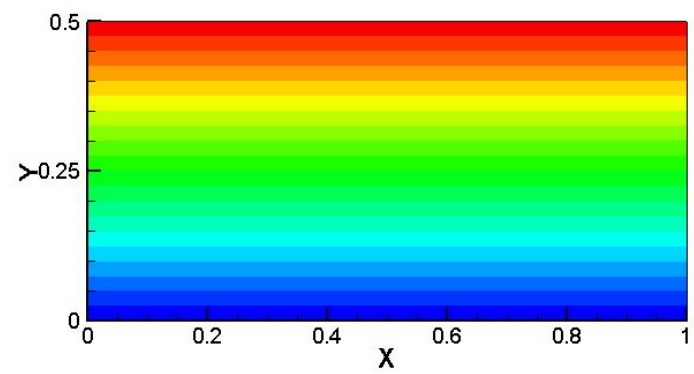

(b)

Figure 6-Streamwise temperature at $\operatorname{Pr}=0.7, \operatorname{Re}=10$ and computational time $t=0.1$ (a) and $t=1(\mathrm{~b})$
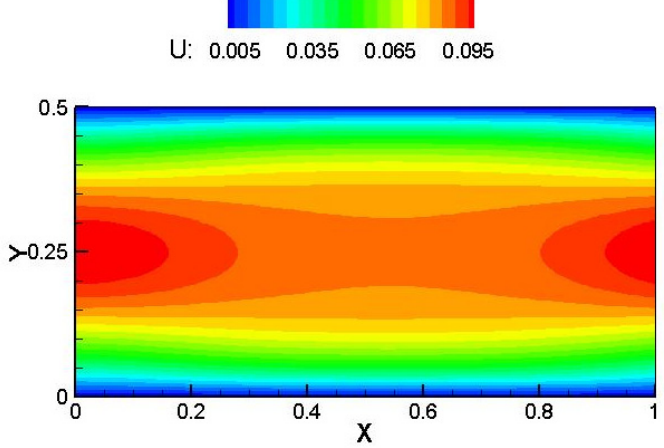

(a)
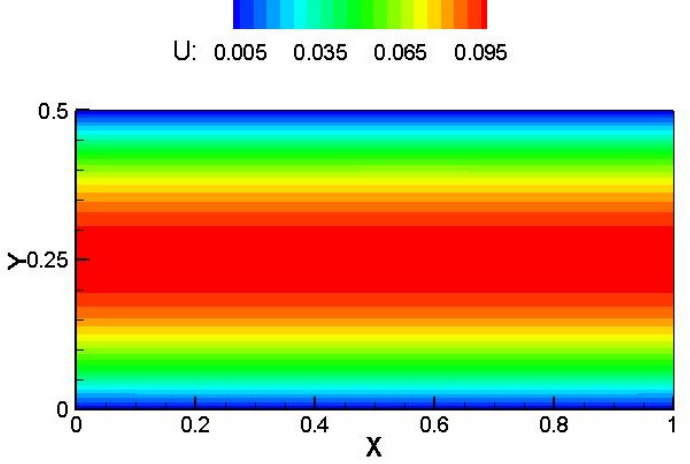

(b)

Figure 7 - Streamwise velocity at $\operatorname{Pr}=0.7, \operatorname{Re}=10$ and computational time $t=0.1$ (a) and $t=1$ (b) 


\section{Conclusion}

The basic aim of this paper is the development of mathematical model for thermal flow in a channel and the implementation of numerical simulation of the problem by the Lattice Boltzmann method applying the D2Q9 model. The validity of this method is tested by comparing the numerical solution to the analytical solution of the planar channel flow. The comparison of the exact solution with the numerical solution for test problem of thermal Poiseuille flow given in Figures 3-5 shows a very good agreement and relationship. It is determined that the numerical method has a second order of accuracy in time. This means that the developed algorithm may well be applied to solving the problem of the dynamic thermal flow in a threedimensional region. This result will be obtained and shown in a future research.

\section{References}

1. Y. Peng, C. Shu, Y.T. Chew, "Simplified thermal lattice Boltzmann model for incompressible thermal flows", Phys. Rev. E 68 (2) (2003) 026701.1-026701.8.

2. A.D'Orazio, M. Corcione, G.P. Celata, "Application to natural convection enclosed flows of a lattice Boltzmann BGK model coupled with a general

purpose thermal boundary condition", Int. J. Therm. Sci. 43 (6) (2004): 575-586.

3. P. Lallemand, L.-S. Luo, "Theory of the lattice Boltzmann method: Acoustic and thermal properties in two and three dimensions", Phys. Rev. E 68 (3) (2003) 036706.1-036706.25.
4. Zhaoli G., Chaguang Z., Baochang S. "Thermal lattice Boltzmann equation for low Mach number flow," Physical Review E 75 (2007): 036704

5. J. Wang, L.-P. Wang, Z. Xiao, Y. Shi, and S. Chen, "A hybrid numerical simulation of isotropic compressible turbulence," J. Comput. Phys. 229, 5257 (2010).

6. Chao Li, Lian-Ping Wang. "An immersed boundary-discrete unified gas kinetic scheme for simulating natural convection involving curved surfaces" Int. J. of Heat and Mass Transfer. (2018): 1059-1070.

7. McNamara G.R., Zanetti G. "Use of the Boltzmann equation to simulate lattice-gas automata" Phys. Rev. Lett. vol. 61, no. 20 (1988): 2332-2335.

8. Chen S., Doolen G.D. "Lattice Boltzmann method for fluid flow" Annu. Rev. Fluid Mech. V. 30. P. 329-364.

9. Nourgaliev R.R., Dinh T.N., Theofanous T.G., Joseph D. "The lattice Boltzmann equation method: theoretical interpretation, numerics and implications" Int. J. of Multiphase Flow vol. 29, no. 1 (2003): 117-169.

10. Kruger, T., Kusumaatmaja, H., Kuzmin, A., Shardt, O., Silva, G., Viggen, E. M. "The Lattice Boltzmann Method." Springer (2017): 694.

11. Portinari M. "2D and 3D verification and validation of the lattice boltzmann method" Diss École Polytechnique de Montréal (2015): 129.

12. C.-H. Liu et al. "Thermal boundary conditions for thermal lattice Boltzmann simulations", Computers and Mathematics with Applications 59 (2010): 2178-2193.

13. F.M. White, "Viscous Fluid Flow," 3rd ed., McGraw-Hill, New York (2006). 